Research letter

\title{
Influence of selenium on clinical and laboratory parameters in patients with rheumatoid arthritis and subclinical hypothyroid dysfunction
}

\author{
Antonina K. Knyazeva ${ }^{1}$, Elena B. Komarova ${ }^{1}$, Natalia N. Kuvichko ${ }^{2}$ \\ ${ }^{1}$ Lugansk State Medical University, Lugansk \\ ${ }^{2}$ Lugansk Regional Clinical Hospital, Lugansk
}

Received 19 February 2016, Revised 25 October 2016, Accepted 4 November 2016

(C) 2016, Knyazeva A.K., Komarova E.B., Kuvichko N.N.

(C) 2016, Russian Open Medical Journal

Abstract: The article presents data on the effectiveness of the impact of selenium on clinical and laboratory parameters of rheumatoid arthritis (RA) in patients with subclinical hypothyroid dysfunction (SHTD).

Aim - to study the influence of selenium on the clinical and laboratory parameters in patients with RA and SHTD.

Material and Methods - The study included 78 patients with RA and SHTD. All patients underwent a complete clinical and functional, instrumental and laboratory examination for RA and thyroid dysfunction. Sodium selenite was added to the standard therapy of rheumatoid arthritis drug.

Results - Our study showed that RA patients with concomitant SHTD after treatment with a complex scheme, which included the preparation of sodium selenite, improved articular syndrome activity of rheumatoid inflammation, reduced levels of thyroid stimulating hormon (TSH) and antithyroidperoxidase (aTPO), reduced the number of patients with elevated levels of aTPO $(p<0.05)$.

Conclusion - The use of sodium selenite in the treatment of RA patients with SHTD is an effective method of correction of the articular syndrome, reducing the levels of markers of inflammation and autoimmune manifestations of the whole process, which is very important and necessary to prevent the progression of the disease.

Keywords: rheumatoid arthritis, subclinical hypothyroid dysfunction, treatment, selenium

Cite as Knyazeva AK, Komarova EB, Kuvichko NN. Influence of selenium on clinical and laboratory parameters in patients with rheumatoid arthritis and subclinical hypothyroid dysfunction. Russian Open Medical Journal 2016; 5: e0406.

Correspondence to Antonina K. Knyazeva. E-mail: antonina-vifanskaya@yandex.ru

\section{Introduction}

One of the important problems of modern rheumatology is the incidence and mortality from cardiovascular disease in patients with rheumatoid arthritis (RA) which is higher than in the general population and associated with a high risk of co-morbidities, including subclinical hypothyroid dysfunction (SHTD), whose development pathogenesis caused chronic generalized rheumatoid inflammation. Having SHTD in RA increases the overall inflammatory activity and, most importantly, increases the characteristic of RA vascular lesions with a more rapid development of endothelial dysfunction (ED) and the subsequent development of atherosclerosis. Urgency and social importance of earlier SHTD and optimal selection of therapy in patients with RA are determined not only by its high prevalence, the affection of the people of working age, a steady progression of the disease in a significant percentage of cases, the rapid development of disability. Among numerous arsenals of therapeutic measures there is no universal method of treating systemic forms of rheumatoid especially against SHTD, and $5-10 \%$ of cases exhibit refractory to any method of treatment. Quite often, a combination of RA and SHTD contributes to further increase of cardiovascular risk [1].

Selenium (Se) is one of the most essential minerals for human health. It has a powerful detoxifying, anti-inflammatory, antiviral effects, inhibits the progression of the acquired immune deficiency syndrome (AIDS) virus infection in the disease, the biochemical carcinogenesis. Following the recommendations of the Academy of Sciences of the United States, to the body of an adult should arrive 50-200 mg Se in a day. It is a part of a series of exogenous proteins, performing both structural and catalytic functions. All Seproteins, whose functions are specifically defined, have strong antioxidant (AO) properties. Among them, a family of enzymes glutathione peroxidase, iodothyronine-diyodinaza, thioredoxin reductase, $\mathrm{P}, \mathrm{O}, \mathrm{W}$ proteins carrying different physiological functions, and etc. Specifically, Se-proteins regulate the level of thyroid hormones, the immune system, spermatogenesis and etc. [2]. AO activity of Se underlies its hepato- and cardioprotective action which is carried out with the participation of glutathione. Se, by virtue of its stock activity, limits or blocks lipid peroxidation - a major risk factor for atherosclerosis, coronary heart disease and other cardiovascular diseases. 
Table 1. Dynamics articular index in patients with RA and SHTD

\begin{tabular}{|c|c|c|c|c|c|c|}
\hline \multirow{2}{*}{ Indicators } & \multicolumn{2}{|c|}{ Igroup $(n=40)$} & \multirow{2}{*}{ p-level } & \multicolumn{2}{|c|}{ II group $(n=38)$} & \multirow{2}{*}{ p-level } \\
\hline & Before treatment & After treatment & & Before treatment & After treatment & \\
\hline Morning stiffness, minutes & $120(100,150)$ & $90(90,105)$ & $<0.001$ & $100(100,130)$ & $90(75,105)$ & 0.002 \\
\hline Number of painful joints & $9(7,12)$ & $5(2,7)$ & $<0.001$ & $7(4,8)$ & $4(2,6)$ & 0.005 \\
\hline Number of swollen joints & $3(2,4)$ & $1(0,2)$ & $<0.001$ & $2(0,3)$ & $1(0,1)$ & 0.006 \\
\hline Index Richie, points & $22(18,23)$ & $8(6,9)$ & 0.001 & $22(19,25)$ & $9(7,11)$ & 0.049 \\
\hline Pain at rest by VAS, $\mathrm{mm}$ & $51(41,58)$ & $35(26,43)$ & $<0.001$ & $45(41,52)$ & $35(28,39)$ & $<0.001$ \\
\hline Pain on movement by VAS, $\mathrm{mm}$ & $53(45,60)$ & $45(37,51)$ & 0.001 & $49(45,57)$ & $43(41,51)$ & 0.060 \\
\hline Health status by $\mathrm{HAQ}$, points & $1.9(1.3,2.2)$ & $1.4(0.9,1.7)$ & 0.004 & $1.8(1.4,2.0)$ & $1.5(1.1,1.8)$ & 0.060 \\
\hline
\end{tabular}

VAS, visual analogue scale; $\mathrm{HAQ}$, health assessment questionnaire. Data presented as Me (LQ, UQ).

Table 2. Dynamics of markers of inflammation in patients with RA and SHTD

\begin{tabular}{|c|c|c|c|c|c|c|}
\hline \multirow{2}{*}{ Indicators } & \multicolumn{2}{|c|}{ I group $(n=40)$} & \multirow{2}{*}{$p$-level } & \multicolumn{2}{|c|}{$1 /$ group $(n=38)$} & \multirow{2}{*}{$p$-level } \\
\hline & Before treatment & After treatment & & Before treatment & After treatment & \\
\hline Rheumatoid factor, IU/mL & $134(48,192)$ & $60(32,128)$ & $<0.001$ & $98(56,164)$ & $72(32,102)$ & $<0.001$ \\
\hline C-reactive protein, $\mathrm{mg} / \mathrm{L}$ & $12.2(9.1,14.1)$ & $8.9(7.5,11.9)$ & $<0.001$ & $9.9(7.3,12.9)$ & $9.0(7.1,11.9)$ & $<0.001$ \\
\hline Erythrocyte sedimentation rate, $\mathrm{mm} / \mathrm{h}$ & $36(30,42)$ & $29(24,34)$ & $<0.001$ & $33(26,36)$ & $29(23,33)$ & $<0.001$ \\
\hline Index DAS28, points & $5.2(5.0,5.9)$ & $4.6(3.9,4.9)$ & $<0.001$ & $4.9(4.1,5.3)$ & $4.2(3.7,4.7)$ & $<0.001$ \\
\hline Sialic acid, units & $260(250,270)$ & $250(240,270)$ & $<0.001$ & $260(240,285)$ & $260(250,270)$ & 0.045 \\
\hline Thymol, units & $3.1(2.3,3.9)$ & $2.9(2.2,3.7)$ & $<0.001$ & $3.1(2.1,3.9)$ & $3.0(2.1,3.9)$ & 0.030 \\
\hline Seromucoid, units & $0.63(0.54,0.70)$ & $0.55(0.43,0.72)$ & 0.001 & $0.6(0.6,0.7)$ & $0.6(0.5,0.7)$ & 0.018 \\
\hline Tumor necrosis factor $\alpha, \mathrm{ng} / \mathrm{L}$ & $65.4(45.5,90.5)$ & $39.5(22.0,58.5)$ & $<0.001$ & $55.4(46.2,70.3)$ & $53.5(46.1,69.5)$ & 0.005 \\
\hline
\end{tabular}

DAS28, Disease Activity Score 28. Data presented as Me (LQ, UQ).

Also, a positive effect of selenium supplementation was ascertained in patients with RA. The use of sodium selenite leads to alleviate symptoms of the disease, improvement of immunological parameters, reduces swelling and morning stiffness of the joints, as well as reducing inflammatory markers, reduces the severity of pain. This is due to anti-oxidant, anti-inflammatory, immunomodulatory effects of sodium selenite [3]. The use of sodium selenite at thyroid dysfunction leads to normalization of thyroid hormone levels, reduces symptoms and improves the health of patients [4]. The findings give a reason to expect a positive clinical effect of sodium selenite in RA patients with concomitant SHTD to improve the underlying disease and the quality of life patients.

\section{Material and Methods}

The study included 78 patients with established diagnosis of RA according to criteria of American College of Rheumatology / European League Against Rheumatism (ACR/EULAR) (2010) with a combination with SHTD established in accordance with international standards for SHTD diagnosis [5]. All patients underwent a complete clinical and functional, instrumental and laboratory examination of rheumatoid arthritis and thyroid dysfunction.

To correct SHTD and effectiveness of the treatment of the underlying disease, we conducted a comprehensive treatment that included use of the sodium selenite at a dose of $200 \mathrm{mg}$ ( 2 tablets in the morning intake during meals) in addition to standard therapy. The therapy started in the hospital. Treatment was carried out for 6 months.

Patients were randomly divided into two groups. Group I $(n=40)$ included patients who received sodium selenite in addition to basic therapy during the 6 months of follow-up. Group II included patients $(n=38)$, who received the standard treatment of RA. The patients included in the two groups matched for age, duration of flow and activity of RA.
Monitoring the effectiveness of the treatment was carried out by studying the dynamics of the follow parameters: number of painful joints (NPJ), number of swollen joints (NSJ), morning stiffness, index Ritchie, visual analogue scale (VAS), health assessment questionnaire (HAQ), rheumatoid factor (RF), Creactive protein, erythrocyte sedimentation rate (ESR) and index Disease Activity Score 28 (DAS28), tumor necrosis factor $\alpha$ (TNF- $\alpha$ ), sialic acid, thymol, seromucoid, thyroid stimulating hormon (TSH) and antithyroidperoxidase (aTPO).

Statistical analysis of the results was performed on a personal (computer using a licensed software package Microsoft Excel (Microsoft, USA) and Statistica v.8.0 (StatSoft Inc., USA). The median (M), lower and upper quartiles (LQ and UQ), MannWhitney criteria, Chi-square ( $\chi 2)$, the accuracy of statistics $(p)$ was evaluated. Statistically significant differences were determined at the level of $p<0.05$. Quantitative data presented as median with interquartile range $-\mathrm{Me}(\mathrm{LQ}, \mathrm{UQ})$.

\section{Results}

Combined therapy contributed to a significant reduction of the articular syndrome. In particular, the group I observed a highly significant decrease in the NPJ $(p<0.001)$, the NSJ $(p<0.001)$ and a significant decrease in the duration of morning stiffness of joints $(p<0.001)$, index Ritchie $(p=0.001)$. The severity of pain at rest and during movement significantly decreased $(p<0.001$ and $p=0.001$ respectively). The health status of $\mathrm{HAQ}$ significantly improved $(p=0.004)$ (Table 1$)$.

In Group II NPJ ( $p=0.005)$ and NSJ ( $p=0.006)$ highly significantly reduced and the duration of morning stiffness $(p=0.002)$, the index Ritchie $(p=0.049)$ and pain at rest VAS $(p<0.001)$ were significantly reduced. We do not get significant motion dynamic pain by VAS and patient's appreciation of life quality by HAQ ( $p=0.060$ and $\mathrm{p}=0.060$ respectively) (Table 1 ). 
Table 3. Dynamics TSH in groups depending on the duration of RA

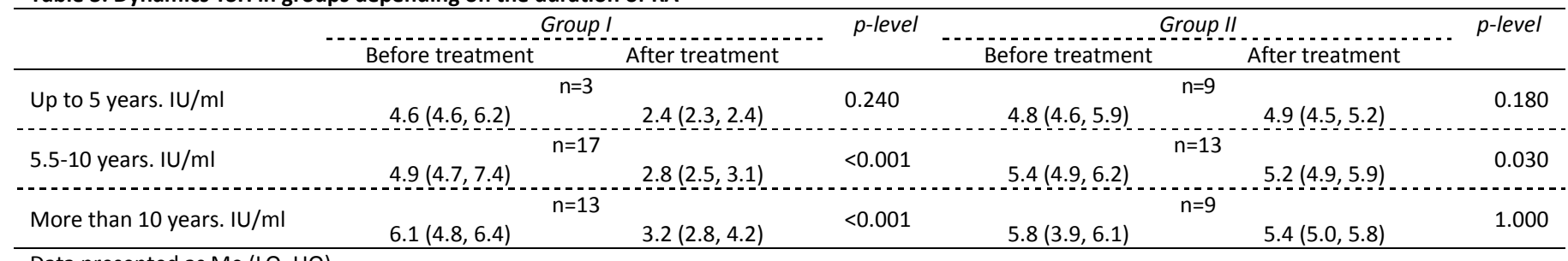

Data presented as Me (LQ, UQ).

Table 4. Dynamics aTPO in groups depending on the duration of RA

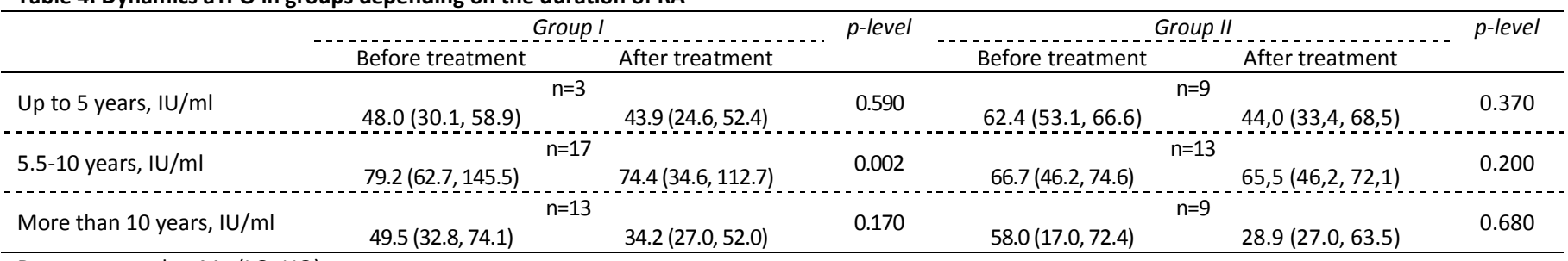

Data presented as $\mathrm{Me}(\mathrm{LQ}, \mathrm{UQ})$.

An analysis of inflammatory markers found that standard therapy and complex treatment resulted in a significant decrease in their levels. Patients in both groups, as in I and II, found highly significant (both $\mathrm{p}<0.001$ ) reduction in levels of rheumatoid factor (RF), C-reactive protein, erythrocyte sedimentation rate (ESR) and index DAS28 (Table 2); probably, almost the same level of reliability, reduction of TNF- $\alpha$ (Table 2 ).

For other studied parameters the positive dynamics in the group I was more significant. In particular, the level of sialic acid and thymol in group I determined a highly significant decrease in the levels after treatment ( $<<0.001$ and $p<0.001$ respectively), and group II likely but with a lower significance level $(p=0.045$ and $\mathrm{p}=0.030$ respectively) (Table 2 ). Comprehensive treatment regimen resulted in a significant reduction in seromucoid than in patients who were treated by the standard procedure $(p=0.001$ and $\mathrm{p}=0.018)$.

There was found highly significant $(p<0.001)$ reduction in the level of TSH from $5.4(4.6,6.6) \mathrm{IU} / \mathrm{I}$ to $2.8(2.5,4.0) \mathrm{IU} / \mathrm{I}$ in patients of group I after the therapy, and in group II, the level of this indicator remained practically unchanged. Its numbers were 5.4 $(4.6,6.2) \mathrm{IU} / \mathrm{I}$ and $5.2(4.7,5.9) \mathrm{IU} / \mathrm{I}$, respectively, at the beginning and at the end of the observation period $(p=0.060)$.

It was found highly significant $(p<0.001)$ aTPO decline from $62.7(38.7,128.4) \mathrm{IU} / \mathrm{ml}$ to $43.9(32.2,89.5) \mathrm{IU} / \mathrm{ml}$ patients in group I. In group II, its levels were $62.4(43.1,74.6) \mathrm{IU} / \mathrm{ml}$ and 52.0 $(32.8,72.1) \mathrm{IU} / \mathrm{ml}$, respectively, at the beginning and at the end of the observation period $(p=0.140)$. It should be noted that after treatment in both groups of patients with RA and SHTD observed reduction in the number of patients with elevated levels aTPO, but it was significant only for patients in group I and insufficiently significant in group II ( $\chi 2=4.59, p=0.032$ vs $\chi 2=1.48, p=0.220)$.

Patients in group I with a duration of RA of 5.5 to 10.0 and over 10 years determined a significant decrease in TSH levels under the influence of the treatment $(p<0.001)$. In group II, a significant decrease in TSH levels was observed only with the duration of RA of 5.5-10.0 years $(p=0.030)$ (Table 3$)$.
We have studied the dynamics of aTPO in both groups before and after treatment, depending on the duration of the RA. Significant reduction aTPO observed with duration of RA of 5.5 to 10.0 years in group I $(p=0.002)$. The results are shown in Table 4.

\section{Discussion}

RA is the most common connective tissue disease [6] and often combined with lesions of the thyroid gland (TG) [7]. SGTD manifested as a "joint syndrome", which has not only the subjective and objective manifestations $[8,9]$

The study showed that RA patients with concomitant SGTD after treatment with a complex scheme, which included the preparation of sodium selenite, improved articular syndrome activity of rheumatoid inflammation, reduced levels of TSH and aTPO, reduced the number of patients with elevated levels of aTPO that probably related to the antioxidant, anti-inflammatory, immunomodulatory effects of sodium selenite [3]. Patients with RA duration of 5.5 to 10 and over 10 years determined a significant decrease in serum TSH and a significant reduction in aTPO with RA duration of 5.5 to 10 years under the influence of complex treatment. At the same time in the group of patients with RA SGTD who received only standard treatment for $\mathrm{RA}$, without the addition of sodium selenite, the outcomes were significantly worse. Of course, the standard treatment of the RA, which includes the use not only nonsteroidal anti-inflammatory drugs (NSAIDs) but also methotrexate, and in some cases, reduces the severity of methylprednisolone articular syndrome, but to a much lesser extent.

Thus, the use of sodium selenite in the treatment of RA patients with SHTD is an effective method of correction of the articular syndrome, reducing the levels of markers of inflammation and autoimmune manifestations of the process as a whole, which is a very important and necessary to prevent disease progression.

\section{Conclusion}

The use of sodium selenite in complex treatment of patients with RA SHTD leads to: a reduction of the articular syndrome, 
manifests a decrease in NPJ, lowers NSJ, duration of morning stiffness of the joints, the index Ritchie, pain intensity at rest and in motion VAS and overall improvements in health by HAQ; positive dynamics of inflammation markers: the RF, TNF- $\alpha$, CRP, ESR, seromucoid, sialic acid and integrative index DAS28; aTPO level reduction by $30 \%$, while reducing the number of patients with elevated aTPO $25 \%$.

\section{Conflict of interest}

No conflict of interest.

\section{References}

1. Nasonov EL. New approaches to pharmacotherapy of rheumatoid arthritis: tofacitinib. Scientific and Practical Rheumatology 2014; 52(2): 209-221. DOI: 10.14412/1995-4484-2014-209-221.

2. Shukurlu $Y$, Mamedov Sh, Huseynov T. Nano impurity regulation of selenium oxidation-destructive processes in biomacromolecules. In: Book of International conference "Fizika 2005". 7-9 June 2005: 583586.

3. Loniewski I. Selen - ein essentielles Spurenelement in der Therapie der rheumatoiden Arthritis. SANUM-Post 2004; 69: 22-24. German

4. Kohrle J, Jakob F, Contempre B, Dumont JE. Selenium, the thyroid, and the endocrine system. Endocrine Reviews 2005; 26(7): 944-984. PMID: 16174820. DOI: 10.1210/er.2001-0034.

5. Surks Ml, Ortiz E, Daniels GH, Sawin $\mathrm{CT}$, Col NF, Cobin RH, et al. Subclinical thyroid disease: scientific review and guidelines for diagnosis and management. JAMA 2004; 291: 228-238. PMID: 14722150. DOI: 10.1001/jama.291.2.228.

6. Kovalenko VN, Bortkevych OP, Bilyavskaya UV. Modern aspects of diagnosis of rheumatoid arthritis. Ukraine Health 2010; 1: 74-77. Ukrainian

7. Raterman HG, Halm VP, Voskuyl AE, Simsek S, Dijkmans BAC, Nurmohamed MT. Rheumatoid arthritis is associated with a high prevalence of hypothyroidism that amplifies its cardiovascular risk. Ann Rheum Dis 2008; 67: 229-232. PMID: 17557891. DOI: 10.1136/ard.2006.068130.

8. Atzeni F, Doria A, Ghirardello A, Turiel M, Batticciotto A, Carrabba M, Sarzi-Puttini P. Anti-thyroid antibodies and thyroid dysfunction in rheumatoid arthritis: prevalence and clinical value. Autoimmunity 2008; 41(1): 111-115. DOI: 10.1080/08916930701620100. PMID: 18176873.

9. Shantha GP, Kumar AA, Jeyachandran V, Rajamanickam D, Rajkumar K, Salim $S$, et al. Association between primary hypothyroidism and metabolic syndrome and the role of $C$ reactive protein: a crosssectional study from South India. Thyroid Res 2009; 2(1): 1-7. PMID: 19272156. DOI: 10.1186/1756-6614-2-2.

Authors:

Antonina K. Knyazeva - PhD, MD, Lugansk State Medical University, Department of Internal Medicine of the Faculty of Graduate Education, Lugansk.

Elena B. Komarova - PhD, MD, Lugansk State Medical University, Department of Internal Medicine of the Faculty of Graduate Education, Lugansk.

Natalia N. Kuvichko - MD, Lugansk regional clinical hospital, Lugansk. 